\title{
Toyama H, Li Y, Hatazawa J, Huang G, Kubota K (eds): PET/CT for inflammatory diseases: basic sciences, typical cases, and review
}

\author{
Singapore: Springer Nature Singapore Pte Ltd., 2020, 233 pages, Hardcover, $169.99 €$
}

\section{Kengo Ito ${ }^{1}$}

Received: 16 March 2020 / Accepted: 16 March 2020 / Published online: 28 March 2020

(c) The Japanese Society of Nuclear Medicine 2020

PET/CT for inflammatory diseases is the culmination of Japan-China nuclear medicine exchange program between the Japanese Society of Nuclear Medicine and the Chinese Society of Nuclear Medicine, and was planned and edited by eminent nuclear medicine physicians in Japan and China. As seen in the headings basic sciences, typical cases, and review, the publication covers aspects from basic science to clinical applications for the use of PET/CT in the field of inflammatory diseases, based on the most recent findings and a large number of case reports.

Currently, FDG PET/CT using ${ }^{18}$ F-FDG is widely used in diagnosing tumors, and more than $95 \%$ of FDG PET/ CT covered by health insurance in Japan is done with the aim of staging tumors or diagnosing recurrences. Recently, FDG PET/CT has been added to the list of diagnostic tools for non-neoplastic diseases, such as cardiac sarcoidosis and large vessel vasculitis, but the number of scans completed in those areas remains low. The increased accumulation of FDG reflects the enhanced glucose metabolism of tumor cells, but since enhanced glucose metabolism is also seen in macrophages and other inflammatory cells, differentiation between inflammation and tumor on FDG PET/CT is an ongoing issue. However, the increased uptake of FDG seen in inflammation is very attractive from the perspective of diagnosing inflammatory disease, and future clinical applications of FDG PET/CT in areas such as identification of inflammatory lesions and determining the level of activity of inflammation are particularly promising.

Sufficient understanding of the utility of FDG PET/CT in inflammatory diseases has not yet been obtained, and we

Kengo Ito

kito@ncgg.go.jp

1 National Center for Geriatrics and Gerontology, Obu, Aichi, Japan cannot say that there is sufficient scientific evidence for its clinical benefit. In Chapter 1 of this book, the most recent findings in basic research on PET/CT related to inflammation using FDG and tracers other than FDG are introduced in multiple reviews by distinguished researchers. This chapter by itself is very well worth reading. It ends with a section titled application of preclinical PET imaging for infectious diseases in which protective clothing during experiments is introduced with pictures. This is deeply pertinent to current circumstances with the rampant spread of the novel coronavirus pneumonia.

Chapter 2 and subsequent chapters provide detailed reviews and case reports on mainly FDG PET/CT for applications in various disease fields [inflammation or fever of unknown origin (IUO and FUO), variety of infectious diseases, hematological diseases mimic inflammation, largevessel vasculitis, rheumatic diseases (collagen diseases), sarcoidosis, and neuroinflammation]. The form of the reviews and case studies is somewhat lacking in consistency, but in addition to the latest reviews, they describe a wide array of cases in Japan and China, including rare cases, which will surely be of interest to readers.

PET/CT for inflammatory diseases is unlike any other resource in bringing together in one volume the present knowledge on PET/CT for inflammatory diseases. It is a highquality textbook written with the cooperation of many doctors and researchers in Japan and China who are active in the field of nuclear medicine. This book will serve as a valuable reference in clinical practice and research for many nuclear medicine physicians and researchers, and I recommend keeping this book on hand as a ready source of information.

Publisher's Note Springer Nature remains neutral with regard to jurisdictional claims in published maps and institutional affiliations. 